\title{
Transplante de células-tronco hematopoéticas e leucemia mieloide aguda: diretrizes brasileiras
}

\section{Hematopoietic stem cells transplantation and acute myeloid leukemia: Brazilian guidelines}

Lucia Mariano R. Silla

Frederico Dulley ${ }^{2}$

Rosaura Saboya ${ }^{2}$

Eduardo Paton ${ }^{3}$

Fabio Kerbauy ${ }^{4}$

Adriano M. Arantes ${ }^{5}$

Nelson Hamerschlak ${ }^{6}$
O objetivo deste trabalho foi definir diretrizes para a indicação do transplante de células-tronco hematopoéticas (TCTH) no tratamento da leucemia mieloide aguda (LMA) no Brasil. O papel do TCTH no tratamento da LMA foi discutido pelos autores e apresentado para a Sociedade Brasileira de Transplante de Medula Óssea na reunião sobre Diretrizes Brasileiras para o TCTH, que o ratificou. Este consenso foi baseado na revisão da literatura internacional e na experiência brasileira em TCTH para o tratamento da LMA. O tratamento ideal para leucemia mieloide aguda em primeira remissão completa (1RC) ainda não está definido. Há consenso na indicação do TCTH alogênico, com condicionamento mieloablativo, para pacientes que apresentem alterações citogenéticas consideradas de alto risco. O TCTH alogênico não está indicado na $1 R C$ para pacientes de baixo risco citogenético e, aparentemente, o TCTH alogênico, autólogo ou a quimioterapia de consolidação são equivalentes para os pacientes de risco intermediário. Rev. Bras. Hematol. Hemoter. 2010;32(Supl. 1):61-65.

Palavras-chave: Transplante de célula-tronco hematopoética; medula óssea; leucemia mieloide aguda.

\section{Introdução: Prevalência e mortalidade da leucemia mieloide aguda (LMA)}

A leucemia mieloide aguda (LMA) representa $90 \%$ dos casos de leucemia aguda no adulto, com idade média ao diagnóstico de 63 anos. Nos EUA, a prevalência de LMA é de 2-3:100.000 habitantes ou de 10.000 a 13.000 casos novos por ano (de novo e secundária) $;^{1}$ destes, apenas 2.000 serão reportados ao Center of International Blood and Marrow Transplant Research (CIBMTR) como submetidos ao transplante de células-tronco hematopoéticas (TCTH). ${ }^{2}$ No Rio Grande do Sul, Brasil, são diagnosticados em todas as idades, a cada ano, 100 casos novos de LMA de novo, com uma incidência de 0.5-1:100.000 habitantes e idade média ao diagnóstico de 42 anos.

Setenta e nove por cento dos casos de LMA de novo ocorrem nos adultos ( $>18$ anos) e, em cinco anos, apenas noventa pacientes (17\%) dos 532 diagnosticados entre 1996 e 2000, estavam vivos, ${ }^{3}$ o que está de acordo com os dados reportados na literatura e que sugere que, fora de ensaios

\footnotetext{
${ }^{1}$ Serviço de Hematologia e Transplante de Medula Óssea, Hospital de Clínicas de Porto Alegre - Porto Alegre-RS.

${ }_{3}^{2}$ Centro de Transplante de Medula Óssea do Hospital das Clínicas da Universidade de São Paulo - São Paulo-SP.

${ }^{3}$ Hemonúcleo do Hospital de Câncer de Barretos - Barretos-SP

${ }_{5}^{4}$ Centro de Transplante de Medula Óssea da Universidade Federal de São Paulo (Unifesp) e Hospital Israelita Albert Einstein - São Paulo-SP.

${ }^{5}$ Serviço de Transplante de Medula Óssea do Hospital Araújo Jorge - Associação de Combate ao Câncer em Goiás - Goiânia-GO.

${ }^{6}$ Programa de Hematologia e Transplante de Medula Óssea do Hospital Israelita Albert Einstein - São Paulo-SP.
}

Programa de Hematologia e Transplante de Medula Óssea do Hospital Israelita Albert Einstein/Instituto Israelita Albert Einstein de Ensino e Pesquisa - São Paulo-SP.

Correspondência: Nelson Hamerschlak

Centro de Pesquisa Clínica, Instituto Israelita de Ensino e Pesquisa Albert Einstein

Av. Albert Einstein, 627/701, Piso Chinuch

05651-901 - São Paulo, SP - Brasil

Tel.: (55 11) 3747-0456 - Fax: (55 11) 3747-0302

E-mail:hamer@einstein.br

Doi: 10.1590/S1516-84842010005000024 
clínicos, e apesar dos avanços do conhecimento nesta área, a LMA ainda é uma doença fatal na imensa maioria dos casos.

\section{Resultados do transplante de célula-tronco hematopoética em LMA - Registro Internacional}

O TCTH alogênico, curativo para a LMA, mas acompanhado de morbimortalidade considerável, está disponível para poucos pacientes; seja pela idade, pela ausência de doadores ou, ainda, pela indisponibilidade de leitos. Dados do CIBMTR $^{4}$ mostram que, até 2006, foram transplantados cerca de 2 mil casos de LMA, por ano, em todos os centros que reportam seus dados àquele registro. Destes, cerca de $50 \%$ foram submetidos ao TCTH alogênico, mieloablativo, aparentado; $40 \%$ receberam transplante mieloablativo não aparentado, e os $10 \%$ restantes receberam TCTH não mieloablativo (a maioria) seguidos pelo TCTH de cordão umbilical. $^{4}$

Segundo Appelbaun, ${ }^{2,5}$ dos 13 mil casos novos de LMA, por ano, nos EUA, considerando centros de transplante não afiliados ao CIBMTR, apenas no máximo 2 mil indivíduos chegam ao TCTH.

Os resultados do TCTH alogênico aparentado com regime de condicionamento mieloablativo para a LMA dependem sobretudo do status da doença: precoce $\left(1^{\mathrm{a}} \mathrm{RC}\right)$, intermediária (em $2^{\mathrm{a}} \mathrm{RC}$ ) ou avançada (em recidiva ou refratária) $61 \%, 50 \%$ e $26 \%$ de sobrevida em três anos, respectivamente, enquanto no TCTH mieloablativo, com doador não aparentado, a sobrevida é de $46 \%$ para precoce e intermediário e $18 \%$ para avançados, no mesmo período de tempo. ${ }^{4}$ Os dados sobre o TCTH em LMA reportados por centros brasileiros ao CIBMTR são muito semelhantes aos do registro. No entanto, é necessário ressaltar que nem todos os casos de LMA em primeira remissão completa (RC) têm indicação de TCTH (dados não mostrados).

\section{Fatores de risco}

Além dos fatores de risco relacionados ao estádio da doença, a estratificação de risco, de acordo com alterações citogenéticas proposta pelo South West Oncology Group $\mathrm{SWOG}^{6}$ e pelo MRC AML 10 Trial $^{7}$ (Tabela 1), é fundamental na definição do tratamento para os diversos subtipos de LMA. Recentemente, uma série de alterações moleculares tem sido relacionada com LMA e estudos estão em andamento no sentido de melhor definir seu papel prognóstico (Tabela 2).

\section{Indicações de TCTH alogênico relacionado na LMA em primeira RC}

Uma meta-análise publicada recentemente, incluindo 6.007 pacientes, mostrou que os resultados do TCTH alogênico relacionado em primeira RC é superior às altas doses de quimioterapia (QT) e ao TCTH autólogo para pacientes de risco intermediário e alto, não estando indicado para pacientes de baixo risco. ${ }^{11}$ É interessante chamar a atenção para o fato de que, dos 1.712 artigos sobre TCTH alogênico em primeira RC para a LMA, identificados naquela meta-análise, 1.660 foram irrelevantes para a definição do tema quando analisados títulos e resumos; dos 52 artigos potencialmente relevantes, apenas 24 puderam ser efetivamente analisados devido ao desenho e análise segundo a intenção de tratamento (ITT intention to treat).

\section{Indicações do TCTH autólogo na LMA em primeira RC}

Embora sucessivas meta-análises tenham demonstrado a não superioridade do TCTH autólogo sobre a quimioterapia em altas doses, ${ }^{12,13,14}$ a experiência brasileira no tratamento da LMA parece indicar um papel para o TCTH autólogo em nosso meio.

Entre os anos de 1996 e 2000, dos 532 pacientes portadores de LMA detectados, a sobrevida em cinco anos foi de $17 \%{ }^{3}$ É importante ressaltar, no entanto, que, neste estudo, dentre os pacientes vivos e em RC após cinco anos, estavam, embora em uma minoria, pacientes submetidos ao TCTH alogênico e pacientes tratados paliativamente ou não tratados.

Por outro lado, em um estudo retrospectivo sobre os resultados do TCTH alogênico e autólogo para LMA no Brasil, ${ }^{15}$ não houve diferença na sobrevida global entre estas duas modalidades de transplante. De maneira que, em nosso meio, o TCTH autólogo é procedimento aceito como consolidação da LMA após dois ciclos de indução e pelo menos um de consolidação para pacientes de risco intermediário sem doador HLA compatível.

Nos pacientes de baixo risco (sem mutação do c-KIT e na presença de NPM1) recomenda-se apenas a QT em altas doses, não havendo indicação de TCTH alogênico ou autólogo. Em casos de citogenética desfavorável, o TCTH alogênico é superior e deve ser indicado sempre que possível.

Na LMA-M3, em segunda RC molecular é aceitável o TCTH autólogo. ${ }^{16} \mathrm{O}$ TCTH alogênico é aceito no tratamento da LMA refratária. ${ }^{17}$

\section{Consolidação e transplante de célula-tronco hematopoética}

O número de consolidações pré-TCTH alogênico nunca foi testado de forma prospectiva. Estudo retrospectivo do CIBMTR com 431 pacientes sugere que não há benefício de QT adicional pós-RC e que o TCTH alogênico deve ser realizado logo que possível. ${ }^{18}$ Para o TCTH autólogo, como ressaltado acima, no Brasil se recomenda pelo menos um ciclo de consolidação pré-transplante. 
Tabela 1. Critérios de estratificação de risco para a LMA

\begin{tabular}{|c|c|c|}
\hline & Critério do SWOG ${ }^{6}$ & $\begin{array}{l}\text { Critério do MRC } \\
\text { (igual ao SWOG exceto:) }\end{array}$ \\
\hline Favorável & $\begin{array}{l}\mathrm{t}(15 ; 17)+\text { qualquer outra anormalidade } \\
\text { inv }(16) / \mathrm{t}(16 ; 16) / \mathrm{del}(16 \mathrm{q})+\text { qualquer outra } \\
\text { anormalidade } \\
\mathrm{t}(8 ; 21) \text { sem del }(9 q) \text { ou cariótipo complexo }\end{array}$ & $\mathrm{t}(8 ; 21)+$ qualquer outra anormalidade \\
\hline Intermediário & $\begin{array}{l}+8,-Y,+6, \text { del }(12 p) \\
\text { cariótipo normal }\end{array}$ & $\begin{array}{l}\text { anorm } 11 q 23 \\
\text { del }(9 q), \text { del }(7 q) \text { - sem outras anormalidades } \\
\text { cariótipo complexo } \\
\text { ( } \geq 3 \text { anormalidades mas }<5 \text { anormalidades) } \\
\text { todas as anormalidades de significado } \\
\text { prognóstico desconhecido }\end{array}$ \\
\hline Desfavorável & $\begin{array}{l}-5 / \text { del }(5 q),-7 / \text { del }(7 q) \\
t(8 ; 21) \text { com del }(9 q) \\
\text { inv }(3 q) \text {, anorm } 11 q 23,20 q . \\
21 q, \text { del }(9 q), t(6 ; 9) \\
T(9: 22) \text {, anorm } 17 p \\
\text { cariótipos complexos ( } \geq 3 \text { anormalidades) }\end{array}$ & 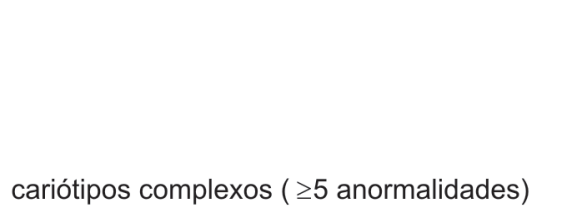 \\
\hline Desconhecido & $\begin{array}{l}\text { Todas as outras aberrações clonais com } \\
<3 \text { anormalidades }\end{array}$ & \\
\hline
\end{tabular}

Tabela 2. Anormalidades moleculares da LMA

\begin{tabular}{|c|c|}
\hline Gene & Características clínicas e biológicas \\
\hline NPM1 & $\begin{array}{l}\text { proteína com funções pleomórficas associada ao sexo feminino } \\
\text { número de blastos (CD33+ e 34- ou baixo) e HDL elevados } \\
25 \% \text { a } 35 \% \text { das LMAs predominantemente com CN (45\%-62\%) associadaa FLT3-ITD e mutação daTKD } \\
\text { genótipo NPM1 mut /FLT3-ITD }{ }^{\text {neg }} \text { associado com bom prognóstico e parece não se beneficiar de TCTH } \\
\text { alogênico mieloablativo. } \\
\text { Quando presente é indicativa de bom prognóstico independentemente do FLT3-ITD status }\end{array}$ \\
\hline FLT3 & $\begin{array}{l}\text { Da família dos receptores de Tirosino-quinase classe III, em investigação para terapia alvo molecular. } \\
\text { FLT3-ITD }{ }^{\text {os }} \text { está associado a mau prognóstico; FLT3-TKD pos é de prognóstico incerto }\end{array}$ \\
\hline ITD & $\begin{array}{l}28 \% \text { a } 34 \% \text { das LMA-CN associada a desfecho desvaforável; a quantificação de ITD parece } \\
\text { importante }\end{array}$ \\
\hline TKD & $\begin{array}{l}\text { Mutação de ponto } 11 \% \text { a } 14 \% \text { das LMA-CN associada a melhor SG. } \\
\text { Altos níveis desta molécula estão associados com melhor SG }\end{array}$ \\
\hline CEBPA & $\begin{array}{l}\text { Fator de transcrição importante na diferenciação de neutrófilos associado principalmente à LMA-CN e } \\
\text { del9q associado a maior taxa de RC, melhor SLR e SG }\end{array}$ \\
\hline$M L L$ & $\begin{array}{l}\text { É uma duplicação "tandem" parcial; } 5 \% \text { a } 11 \% \text { das LMA-CN associada à menor duração da RC ou à } \\
\text { SLR ou SLE menor } \\
\text { TCTH autólogo parece ter um papel favorável nos desfechos }\end{array}$ \\
\hline RAS & Mutação NRAS encontrada em 9\% das LMA-CN sem significado prognóstico \\
\hline WT1 & $\begin{array}{l}\text { mutação encontrada em } \sim 10 \% \text { das LMA-CN. Sozinha, esta mutação não parece ter impacto em } \\
\text { desfechos, mas se associada ao genótipo WT1mut } / \text { FLT3-ITD pos eleva o risco de falência na indução } 10\end{array}$ \\
\hline
\end{tabular}

ITD - internal tandem duplication; TKD - mutação de domínio da tirosino-quinase; CN - cariótipo normal; RCremissão completa; SG - sobrevida global; SLR - sobrevida livre de recaída; SLE - sobrevida livre de eventos. Modificado de Döhner $\mathrm{H}^{8}$

\section{Enxerto de medula óssea (MO) ou sangue periférico (SP)}

Em uma meta-análise incluindo nove estudos randomizados, incluindo 1.111 pacientes, ficou demonstrado que o TCTH de sangue periférico (SP) é superior ao de medula óssea (MO) por levar a uma diminuição da taxa de recidiva, melhor sobrevida global (SG) e sobrevida livre de doenças (SLD). ${ }^{19}$

É importante ressaltar, no entanto, que o TCTH de SP está associado com um risco significativo de doença do enxerto versus hospedeiro extensa, devendo ficar reservado a pacientes com doença avançada $\left(\geq 2^{\mathrm{a}} \mathrm{RC}\right)$. 


\section{Regimes de condicionamento}

A comparação entre bussulfano + ciclofosfamida $(\mathrm{Bu}-$ $\mathrm{CY})$ e bussulfano + irradiação corporal total (Bu-TBI) realizada em 581 pacientes (381 condicionados com $\mathrm{Bu}-\mathrm{CY}$ e 200 com Bu-TBI) mostrou que, embora o Bu-TBI pareça estar relacionado a uma diminuição na recidiva, não foram observadas diferenças significativas entre mortalidade relacionada ao transplante (MRT), sobrevida livre de doença (SLD) e SG. ${ }^{20}$ Mais recentemente, a associação de fludarabina a bussulfano (Flu-Bu) tem sido empregada e parece estar associada a menor toxicidade e eficácia equivalente ao $\mathrm{Bu}-\mathrm{CY}$ em comparação histórica. ${ }^{21}$ Embora resultados promissores e semelhantes com este regime de condicionamento tenham sido observados no Brasil, pela Conexão Caipira (REF), é importante ressaltar que o protocolo original foi descrito com a utilização de bussulfano intravenoso, não disponível em nosso meio. Baseados nestas evidências, é aceitável no Brasil o emprego destas três modalidades de condicionamento: $\mathrm{Bu}-\mathrm{Cy}$, Flu-Bu e $\mathrm{Bu}-\mathrm{TBI}$ a ser definido de acordo com a experiência de cada grupo e disponibilidades.

\section{TCTH alogênico não aparentado}

Com exceção de um estudo que arrolou um pequeno número de pacientes, ${ }^{22}$ não existem estudos prospectivos comparando o TCTH alogênico não aparentado com o aparentado. Os resultados das análises retrospectivas sugerem que o TCTH alogênico não aparentado é uma opção para o paciente sem doador HLA idêntico na família. Com as técnicas moleculares mais sensíveis para a tipagem HLA, os resultados do TCTH não aparentado parecem ser semelhantes ao aparentado para os pacientes portadores de LMA de alto risco $^{23}$

Quanto à fonte de células, os resultados parecem ser semelhantes com a utilização de doadores não aparentados de MO ou sangue de cordão umbilical. ${ }^{24}$

\section{Recomendações finais}

Para a melhoria dos resultados e para substanciar as diversas modalidades de TCTH para o tratamento da LMA, no Brasil, deve-se realizar um esforço nacional para a cariotipagem de qualidade assim como para o desenvolvimento de técnicas moleculares, ambos instrumentais para a estratificação de risco e o tratamento ideal para a LMA.

Resumidamente, o consenso com as respectivas graduações de qualidade de evidência e força de recomendação foi:

- O TCTH autólogo é procedimento aceito no tratamento de consolidação das leucemias mieloides agudas após dois ciclos de indução e pelo menos um de consolidação (B2).
- Em casos de mau prognóstico (citogenética desfavorável), o transplante alogênico é superior e deve ser indicado sempre que possível (A1). Parece também superior em casos de prognóstico intermediário (B2). É aceito em casos de leucemia refratária $(\mathrm{C} 2)$.

- O TCTH autólogo é procedimento aceito no tratamento de consolidação das leucemias mieloides agudas após dois ciclos de indução e pelo menos um de consolidação (B2). A experiência brasileira sugere que o TCTH autólogo é superior à consolidação apenas com quimioterapia $(\mathrm{C} 2)$.

- O TCTH autólogo, apesar de piores resultados, pode ser indicado na consolidação em casos de leucemia secundária na ausência de doadores alogênicos e apresenta piores resultados quanto mais avançada a doença $\left(1^{\text {a }}\right.$ remissão $>2^{\mathrm{a}}$ remissão $>$ doença avançada $(\mathrm{C} 2)$.

- Na LMA M3, o TCTH autólogo é aceito em segunda remissão completa molecular $(\mathrm{C} 2)$.

- Não há benefício de quimioterapia adicional pósremissão completa antes do transplante alogênico, que deve ser realizado logo que possível. Portanto, o TCTH alogênico logo após a RC. TCTH autólogo após pelo menos uma consolidação (B1).

- Em pacientes com doença avançada, o sangue periférico parece ser melhor (C2).

- Esquemas de condicionamento recomendados: $\mathrm{Bu}-$ Cy/Bu-Mel, Flu-Bu, TBI-Cy. Em cordão umbilical considerar esquemas baseados com ATG (B1).

\begin{abstract}
The objective of this work was to define guidelines for the indication of hematopoietic stem cells transplantation (HSCT) in the treatment of acute myeloid leukemia (AML) in Brazil. The role of HSCT in the treatment of AML was discussed by the authors and presented to the Brazilian Society of Bone Marrow Transplantation in a meeting to formulate and ratify the Brazilian Guidelines on HSCT. This consensus was based on a review of international publications and on the Brazilian experience in HSCT for the treatment of AML. The optimal treatment for AML in first complete remission (1CR) has not been defined yet. There is consensus on the indication of allogeneic HSCT with myeloablative conditioning for patients who present high risk cytogenetic changes. Allogeneic HSCT is not indicated for low cytogenetic risk $1 R C$ patients and, apparently, allogeneic and autologous HSCT and consolidation chemotherapy are similar for intermediate risk patients Rev. Bras. Hematol. Hemoter. 2010;32 (Supl. 1):61-65.
\end{abstract}

Key words: Hematopoietic stem cell transplantation; bone marrow; acute myeloid leukemia. 


\section{Referências Bibliográficas}

1. Jemal A, Thomas A, Murray T, Thun M. Cancer statistics 2002. CA Cancer J Clin. 2002;52(1):23-47.

2. Appelbaum FR. Allogeneic hematopoietic cell transplantation for acute myeloid leukemia when a matched related donor is not available. Hematology Am Soc Hematol Educ Program. 2008:412-7.

3. Capra M, Vilella L, Pereira WV, et al. Estimated number of cases, regional distribution and survival of patients diagnosed with acute myeloid leukemia between 1996 and 2000 in Rio Grande do Sul, Brazil. Leuk Lymphoma. 2007;48(12):2381-6.

4. Center for International Blood and Marrow Transplant Research https://campus.mcw.edu/AngelUploads/Content/CS IBMTR2/ _as soc/ECCBED0 AF 0 A 4492B B 667FB6227DC7C06/ SummarySet06 Pt2 files/frame.htm - acessado em 15/07/2009.

5.Appelbaum FR. Optimising the conditioning regimen for acute myeloid leukaemia. Best Pract Res Clin Haematol. 2009, 22(4): 543-50.

6. Slovak ML, Kopecky KJ, Cassileth PA, et al. Karyotypic analysis predicts outcome of preremission and postremission therapy in adult acute myeloid leukemia: a Southwest Oncology Group/ Eastern Cooperative Oncology Group study. Blood. 2000;96 (13):4075-83.

7. Grimwade $\mathrm{D}$, Walker $\mathrm{H}$, Oliver $\mathrm{F}$, et al. The importance of diagnostic cytogenetics on outcome in AML: analysis of 1,612 patients entered into the MRC AML 10 trial. The Medical Research Council Adult and Children's Leukaemia Working Parties. Blood. 1998; 92(7):2322-33.

8. Döhner H. Implication of the molecular characterization of acute myeloid leukemia. Hematology Am Soc Hematol Educ Program. 2007:412-9.

9. Schneider F, Hoster E, Unterhalt M, Schneider S, et al. NPM1 but not FLT3-ITD mutations predict early blast cell clearance and CR rate in patients with normal karyotype AML(NK-AML) or high-risk myelodysplastic syndrome (MDS). Blood. 2009;113 (21):5250-53.

10. Gaidzik VI, Schlenk RF, Moschny S, et al. Prognostic impact of WT1 mutations in cytogenetically normal acute myeloid leukemia: a study of the German-Austrian AML Study Group. Blood. 2009; 113(19):4505-11.

11. Koreth J, Schlenk R, Kopecky KJ, et al. Allogeneic stem cell transplantation for acute myeloid leukemia in first complete remission systematic review and meta-analysis of prospective clinical trials. JAMA. 2009;301(22):2349-61.

12. Nathan PC, Sung L, Crump M, Beyene J. Consolidation therapy with autologous bone marrow transplantation in adults with acute myeloid leukemia: a meta-analysis. J Natl Cancer Inst. 2004; 96(1):38-45.

13. Levi I, Grotto I, Yerushalmi R, Ben-Bassat I, Shpilberg O. Metaanalysis of autologous bone marrow transplantation versus chemotherapy in adult patients with acute myeloid leukemia in first remission. Leuk Res. 2004 (6):605-12.

14. Breems DA, Boogaerts MA, Dekker AW et al. Autologous bone marrow transplantation as consolidation therapy in the treatment of adult patients under 60 years with acute myeloid leukaemia in first complete remission: a prospective randomized Dutch-Belgian Haemato-Oncology Co-operative Group (HOVON) and Swiss Group for Clinical Cancer Research (SAKK) trial. Br J Haematol. 2005;128(1):59-65.

15. Hamerschlak N, Barton D, Pasquini R, Sarquis YN, Ferreira E, Moreira FR et al. Estudo retrospectivo do tratamento de leucemia mielóide aguda com o transplante de medula óssea - A experiência brasileira. Rev Bras Hematol Hemoter. 2006;28(1):11-8.
16. Thomas X, Dombret H, Cordonnier C, et al. Treatment of relapsing acute promyelocytic leukemia by all-trans retinoic acid therapy followed by timed sequential chemotherapy and stem cell transplantation. APL Study Group. Acute promyelocytic leukemia. Leukemia. 2000;14(6):1006-13.

17. Fung HC, Stein A, Slovak M, Odonnell MR, et al. A long-term follow-up report on allogeneic stem cell transplantation for patients with primary refractory acute myelogenous leukemia: impact of cytogenetic characteristics on transplantation outcome. Biol Blood Marrow Transplant. 2003;9(12):766-71.

18. Tallman MS, Rowlings PA, Milone G, et al. Effect of postremission chemotherapy before human leukocyte antigen-identical sibling transplantation for acute myelogenous leukemia in first complete remission. Blood. 2000;96(4):1254-8.

19. Stem Cell Trialists Collaborative Group. Allogeneic peripheral blood stem-cell compared with bone marrow transplantation in the management of hematologic malignancies: an individual patient data meta-analysis of nine randomized trials. J Clin Oncol. 2005; 23(22):5074-87.

20. Litzow MR, Pérez WS, Klein JP et al. Comparison of outcome following allogeneic bone marrow transplantation with cyclophosphamide-total body irradiation versus busulphan-cyclophosphamide conditioning regimens for acute myelogenous leukaemia in first remission. Br J Haematol. 2002;119(4): 1115-24.

21. de Lima M, Couriel D, Thall PF, et al. Once-daily intravenous busulfan and fludarabine: clinical and pharmacokinetic results of a myeloablative, reduced-toxicity conditioning regimen for allogeneic stem cell transplantation in AML and MDS. Blood. 2004;104(3):857-64.

22. Krauter J, Heil G, Hoelzer D, et al. Role of consolidation therapy in the treatment of patients up to 60 years with high risk AML. Blood. 2005;106:172 (abstract).

23. Appelbaum FR. Hematopoietic cell transplantation from unrelated donors for treatment of patients with acute myeloid leukemia in first complete remission. Best Pract Res Clin Haematol. 2007;20(1):67-75.

24. Rocha V, Labopin M, Sanz G, et al. Transplants of umbilical-cord blood or bone marrow from unrelated donors in adults with acute leukemia. N Engl J Med. 2004;351(22):2276-85.

Este artigo foi avaliado pelos coordenadores das Diretrizes do Transplante de Medula Óssea da Sociedade Brasileira de Transplante de Medula Óssea, Luis Fernando Bouzas, Prof. Julio Cesar Voltarelli e Nelson Hamerschlak, e publicado após avaliação e revisão do editor, Milton Artur Ruiz.

Conflito de interesse: sem conflito de interesse

Recebido: 05/11/2009

Aceito: 23/11/2009 